\title{
Optimal Insurance Design Under a Value-at-Risk Framework
}

CHING-PING WANG*

cpwang@isu.edu.tw

Department of Finance, I-Shou University, No. 1, Section 1, Hsueh-Cheng Rd., Ta-Hsu Hsiang,

Kaohsiung County, Taiwan

DAVID SHYU

dshyu@cm.nsysu.edu.tw

Department of Finance, National Sun Yat-Sen University, No. 70, Lien-Hai Rd., Kaohsiung, Taiwan

HUNG-HSI HUANG

d86723002@ntu.edu.tw

Department of Business Administration, Southern Taiwan University of Technology, No. 1, Nan-Tai Street,

Yung-Kang, Taiwan

Received February 23, 2004; Revised December 16, 2004

\begin{abstract}
This study designs an optimal insurance policy form endogenously, assuming the objective of the insured is to maximize expected final wealth under the Value-at-Risk (VaR) constraint. The optimal insurance policy can be replicated using three options, including a long call option with a small strike price, a short call option with a large strike price, and a short cash-or-nothing call option. Additionally, this study also calculates the optimal insurance levels for these models when we restrict the indemnity to be one of three common forms: a deductible policy, an upper-limit policy, or a policy with proportional coinsurance.
\end{abstract}

Key words: value at risk, optimal insurance, deductible, policy limit, coinsurance

JEL Classification No.: $\quad$ G22

\section{Introduction}

This study focuses on deriving the optimal insurance policy form endogenously under a value-at-risk (VaR) framework, where VaR is defined as the worst expected loss over a given horizon at a given confidence level (Jorion [2001]). In this framework, the insured's objective is to maximize expected final wealth, but has a VaR constraint that must be met. In addition to deriving the optimal insurance, this study also calculates the optimal insurance levels for three alternative insurances in which we restrict the indemnity to be one of three common forms: a deductible policy, an upper-limit policy, or a policy with proportional coinsurance.

At least three reasons exist why this study addresses the problem of the optimal insurance policy under a VaR framework. First, institutions frequently face different forms of risk, 
most of which can be insured, and VaR has been adopted by institutions around the world as a risk management tool. ${ }^{1}$ It is natural for institutions facing different forms of risk to purchase risk reducing insurance. For example, insurance policies now can be purchased to protect against losses such as human error, loss of staff, interruption of business, trading losses, technology failure, and so on. Second, every individual always faces the risk of uncertain future wealth and income. Like institutions, individuals can adopt VaR for risk management. Naturally, individuals can purchase insurance to control VaR. Third, VaR has progressively been adopted as the asset allocation constraint, for example, Campbell et al. [2001] and Basak and Shapiro [2001]. ${ }^{2}$ Additionally, given a fixed confidence level, although bankers are frequently asked to calculate their $\mathrm{VaR}$ in order to determine their regulatory capital, however, bankers can also retain the current capital level and adjust their asset allocation, or can simply purchase insurance to meet their VaR criteria. Restated, bankers can meet both the given regulatory confidence level and VaR by purchasing insurance.

Various investigations have examined optimal insurance policy design. These investigations can be divided into two frameworks, with and without using expected utility. Under the expected utility framework, Arrow [1974], and Raviv [1979] derived the optimal policy form endogenously. Their studies assumed nonnegative indemnity and no moral hazard, but subsequent studies relaxed these assumptions. Huberman, Mayers, and Smith [1983] focused on two insurance policies, upper limits on coverage and deductibles, and incorporated moral hazard into their analysis. Next, Gollier [1987] relaxed the constraint that insurance payments are always nonnegative to analyze optimal insurance contracts. Under the non-expected utility framework, Doherty and Eeckhoudt [1995] adopted Yaari's Dual Theory to derive the optimal insurance. Since expected utility is linear in probability but nonlinear in wealth, and since Yarri's Dual Theory is linear in wealth but nonlinear in probability, Yaari's Dual Theory generally obtains better results than expected utility theory. Next, using the framework of second-degree stochastic dominance, Gollier and Schlesinger [1996] provided a new proof for the optimality of deductible insurance, and Schlesinger [1997] examined insurance policy goodness.

Similar to most previous research, this study endogenously derives the optimal insurance policy form. However, unlike previous research, this study designs an optimal insurance under a VaR framework. Like Doherty and Schlesinger [1983a], Doherty and Eeckhoudt [1995], Li and Liu [2003], and Gollier [2003], this study assumes the insurance loading to be proportional to expected indemnity. The main contents of this study include the following: First, this study respectively derives the optimal insurance and the three alternative insurances without making any assumption regarding the probability density function of the risk. Second, to obtain more explicit indemnity schedules for all these insurance forms, this study further assumes that risk is uniformly distributed and lognormally distributed.

Section 2 derives the optimal insurance and alternative insurances under a VaR constraint. Sections 3 and 4 then present the optimal insurance and alternative insurances under the uniform and lognormal distributions, respectively. Finally, Section 5 presents conclusions. 


\section{The model}

\subsection{Assumptions}

An insured has an initial wealth, $W_{0}$, and faces a risk of loss, $X$, which is a non-negative continuous random variable with probability density function, $f(x)$ and cumulative distribution function, $F(x)$. An insurance policy can reduce this risk. The insurance policy costs a premium, $P$, and pays an indemnity schedule, $I(x), 0 \leq I(x) \leq x$ for all $x$. Assume the insurer is risk neutral and the premium accordingly has the form $P=(1+\lambda) \bar{I}$, where $\bar{I}$ and $\lambda$ denote the expected indemnity payment and percentage loading, respectively. That is, the insurance cost is proportional to the expected indemnity payment and the loading fee is $\lambda \bar{I}$. Consequently, the insured's final wealth is $W=W_{0}-P-X+I(X)$ and expected final wealth is $\bar{W}=W_{0}-P-\bar{x}+\bar{I}$, where $\bar{x}$ denotes the expected loss.

This study assumes that the objective of the insured is to choose $I(x)$ to maximize the expected final wealth under the VaR constraint, where VaR is the worst expected loss over a given horizon at a given confidence level. Mathematically, the VaR constraint means $\operatorname{Pr}\{W \geq \bar{W}-v\}=1-\alpha$, where $v \equiv \mathrm{VaR}, 1-\alpha=$ the confidence level, and $\alpha=$ the significance level.

\subsection{Optimal insurance policy design}

This subsection designs the optimal insurance that is Pareto-efficient, i.e. first-best optimal. ${ }^{3}$ The optimal insurance form with the indemnity schedule, $I(x)$, must sufficiently satisfy the insured's objective and meet the premium request of the insurer. According to the previous subsection, the optimal $I(x)$ can be obtained as follows:

$$
\begin{array}{ll}
\underset{I(x)}{\operatorname{Maximize}} & \bar{W}=W_{0}-P-\bar{x}+\bar{I} \\
\text { Subject to } & \operatorname{Pr}\{W \geq \bar{W}-v\} \geq 1-\alpha \quad \text { and } \quad P=(1+\lambda) \bar{I}
\end{array}
$$

Using equalities $P=(1+\lambda) \bar{I}$ and $W=W_{0}-P-X+I(X), \operatorname{Pr}\{W \geq \bar{W}-v\} \geq 1-\alpha$ can be rewritten as

$$
\operatorname{Pr}\{X-I(X) \leq v+\bar{x}-\bar{I}\} \geq 1-\alpha .
$$

Substituting $P=(1+\lambda) \bar{I}$ into eqaution (1) yields $\bar{W}=W_{0}-\bar{x}-\lambda \bar{I}$. Since $W_{0}, \bar{x}$, and $\lambda$ do not depend on $I(x)$, eqaution (1) means to minimize the indemnity to reduce the loading fee. Accordingly, the optimality problem can be restated as follows.

$$
\underset{I(x)}{\operatorname{Minimize}} \bar{I}
$$

Subject to equation (2)

Minimizing $\bar{I}$ is equivalent to maximizing $\bar{x}-\bar{I}$, since $\bar{x}$ does not depend on $I(x)$. Consequently, the insured sets $I(x)$ to minimize loading fee. In fact, $x-I(x)$ represents the 
retention of the loss, and thus $\bar{x}-\bar{I}$ represents the expected retention. The constraint indicates the probability that the retention does not exceed the particular critical value, $\hat{x} \equiv v+\bar{x}-\bar{I}$, at least equals the confidence level $(1-\alpha)$.

Since equation (3) is difficult to solve directly, like Raviv [1979] and Gollier and Schlesinger [1996], we divide the optimality problem into two sub-problems. First, the premium $P$ is assumed fixed and the form of the optimal insurance coverage is found as a function of $P$. Second, the optimal $P$ is chosen. Since $P=(1+\lambda) \bar{I}$, the fixed value of $P$ is equivalent to fixed $\bar{I}$.

Considering a fixed value of $\bar{I}$, the first sub-problem is to choose $I(x)$, which must satisfy the restriction of $E[I(X)]=\bar{I}$ and minimize the probability $\operatorname{Pr}\{X-I(X)>v+\bar{x}-\bar{I}\}$. To save the loading fee, we start considering no indemnity paid yet. That is, when $\bar{I}=0$ and then the corresponding indemnity schedule $I(x) \equiv 0$ for all $x$. Substituting $\bar{I}=0$ and $I(x) \equiv 0$ into equation (2), the constraint is changed to

$$
\operatorname{Pr}\{X \leq v+\bar{x}\} \geq 1-\alpha
$$

Accordingly, we obtain

$$
\text { If } \operatorname{Pr}\{X \leq v+\bar{x}\} \geq 1-\alpha, \quad \text { then } \quad I(x) \equiv 0 \quad \text { for all } x .
$$

This study starts to consider another case that does not satisfy equation (4), restated, the insured requires insurance and thus $\bar{I}>0$. Additionally, the constraint of equation (2) must be binding for optimality, since $X$ has a continuous probability distribution. Accordingly, equation (3) can be rewritten as follows.

$$
\begin{array}{cl}
\underset{I(x)}{\operatorname{Minimize}} & \bar{I} \\
\text { Subject to } & \operatorname{Pr}\{X-I(X) \leq v+\bar{x}-\bar{I}\}=1-\alpha
\end{array}
$$

Equation (7) is equivalent to $\operatorname{Pr}\{X-I(X)>v+\bar{x}-\bar{I}\}=\alpha$. Fixing a value of $\bar{I}>0$, $I(x)$ must be chosen to minimize the probability $\operatorname{Pr}\{X-I(X)>v+\bar{x}-\bar{I}\}$. For losses such that $x>v+\bar{x}-\bar{I}$, an indemnity can be paid to bring this to an equality, that is, $I(x)=x-(v+\bar{x}-\bar{I}) .{ }^{4}$ However, this might violate the restriction that $E[I(X)]=\bar{I}$. To save loading fees, the insured sub-interval is selected on the smaller part of $x>v+\bar{x}-\bar{I}$. The insured sub-interval is assumed to be $v+\bar{x}-\bar{I}<x<A$, where $A$ is a particular critical value. Since $X$ is continuous, $A$ is unique. In sum, in the case where $\operatorname{Pr}\{X>v+\bar{x}\}>\alpha$, the optimal indemnity schedule $I(x)$ is summarized as follows.

$$
\text { If } \operatorname{Pr}\{X>v+\bar{x}\}>\alpha, \quad \text { then } I(x)= \begin{cases}0 & x \leq \hat{x} \\ x-\hat{x} & \hat{x}<x<A \\ 0 & x \geq A\end{cases}
$$

Since $\operatorname{Pr}\{X-I(X)>v+\bar{x}-\bar{I}\}$ is increasing in $\bar{I}$. If we now increase (decrease) $\bar{I}$, the minimum probability will fall (rise). Consequently, the second sub-problem is to adjust 
$\bar{I}$ such that the probability equals the value $\alpha$. From equation (8), we can further obtain that

$$
\operatorname{Pr}\{X-I(X)>v+\bar{x}-\bar{I}\}=\operatorname{Pr}\{X \geq A\}=1-F(A)=\alpha
$$

Equation (9) implies $A=F^{-1}(1-\alpha)$, where $F^{-1}(\cdot)$ denotes the inverse function of $F$. Finally, $\bar{I}$ must be adjusted to satisfy the following equation:

$$
\bar{I}=E[I(X)]=\int_{\hat{x}}^{A}(x-\hat{x}) f(x) d x, \quad \hat{x} \equiv v+\bar{x}-\bar{I} .
$$

Actually, the condition $\operatorname{Pr}\{X>v+\bar{x}\}>\alpha$ is equivalent to $A>v+\bar{x}$, since $A=$ $F^{-1}(1-\alpha)$. Combining this with equations (5), (8), (9), and (10), the optimal indemnity schedule is summarized as follows:

$$
I(x \mid A \leq v+\bar{x}) \equiv 0 \quad \text { for all } \mathrm{x}
$$

and

$$
I(x \mid A>v+\bar{x})= \begin{cases}0 & x \leq \hat{x} \\ x-\hat{x} & \hat{x}<x<A . \\ 0 & x \geq A\end{cases}
$$

Additionally, the optimal indemnity schedule is listed in Table 1. Moreover, figure 1 indicates the probability density function of loss and shows the optimal indemnity schedule. To analyze the retained loss, this study draws the probability density function of retention $(x-I(x))$ in figure 2. Especially, the retained loss has a probability mass $(=F(A)-F(\hat{x}))$ at $\hat{x}$. Figures 3 and 4 respectively illustrate the relations of indemnity and retention versus loss.

Interestingly, the optimal indemnity schedule can be decomposed as three options. From equation (12), we obtain

$$
I(x)=\operatorname{Max}\{x-\hat{x}, 0\}-\operatorname{Max}\{x-A, 0\}-(A-\hat{x}) \times 1_{x \geq A},
$$

where $1_{x \geq A}$, an index function, equals 1 if $x \geq A$ and otherwise equals 0 . Using $x$ as the underlying asset and taking the same maturity as the insurance policy, $\operatorname{Max}\{x-\hat{x}, 0\}$ and

Table 1. Optimal indemnity schedule.

\begin{tabular}{lllll}
\hline Case & $A \leq v+\bar{x}$ & $A>v+\bar{x}$ & \\
\hline$x$ interval & $\forall x$ & $0<x<\hat{x}$ & $\hat{x}<x<A$ & $A<x$ \\
$I(x)$ & $I(x)=0$ & $I(x)=0$ & $I(x)=x-\hat{x}$ & $I(x)=0$ \\
$x-I(x)$ & $x$ & $x$ & $\hat{x}$ & $x$
\end{tabular}




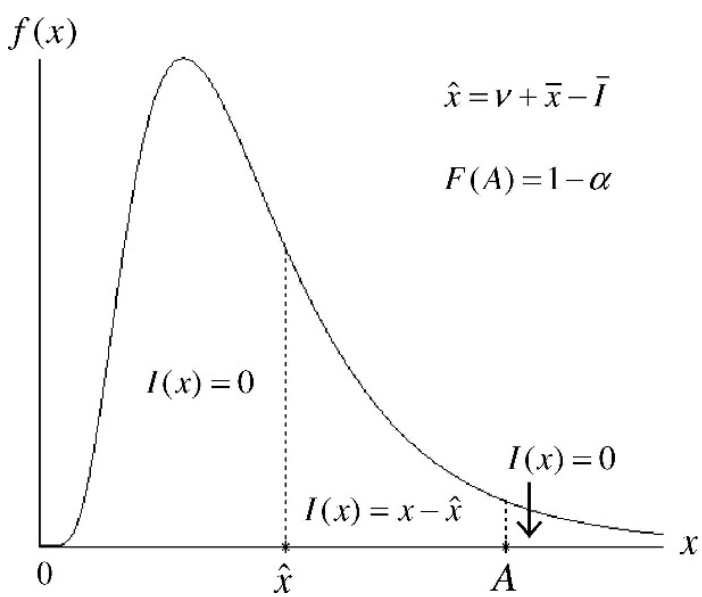

Figure 1. Probability density function of loss and optimal indemnity schedule.

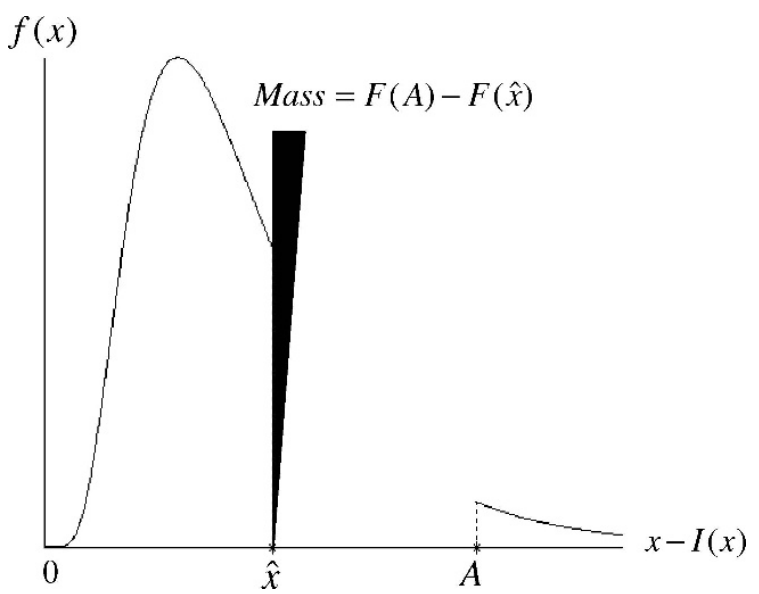

Figure 2. Probability density function of retention.

$\operatorname{Max}\{x-A, 0\}$ are the call options with strike prices $\hat{x}$ and $A$, respectively. By definition, a cash-or-nothing call option pays off nothing if the asset price ends up below the strike price at maturity, and pays a fixed amount if it ends up above the strike price (Hull [2003] p. 441). Taking $A$ as the strike price and $A-\hat{x}$ as the fixed amount, then $(A-\hat{x}) \times 1_{x \geq A}$ is exactly equivalent to the payoff of a cash-or-nothing call option. In short, the optimal indemnity schedule is the same as a long call option with a small strike price, a short call option with a large strike price, and a short cash-or-nothing call option.

From equations (9) and (10), the main comparative static results can be derived as below, with the proofs being presented in Appendixes 1 and 2, respectively.

$$
\partial^{2} \bar{I} / \partial \nu^{2}>0>\partial \bar{I} / \partial \nu \quad \text { and } \quad \partial^{2} \bar{I} / \partial \alpha^{2}>0>\partial \bar{I} / \partial \alpha \quad \text { if } A>\hat{x} .
$$




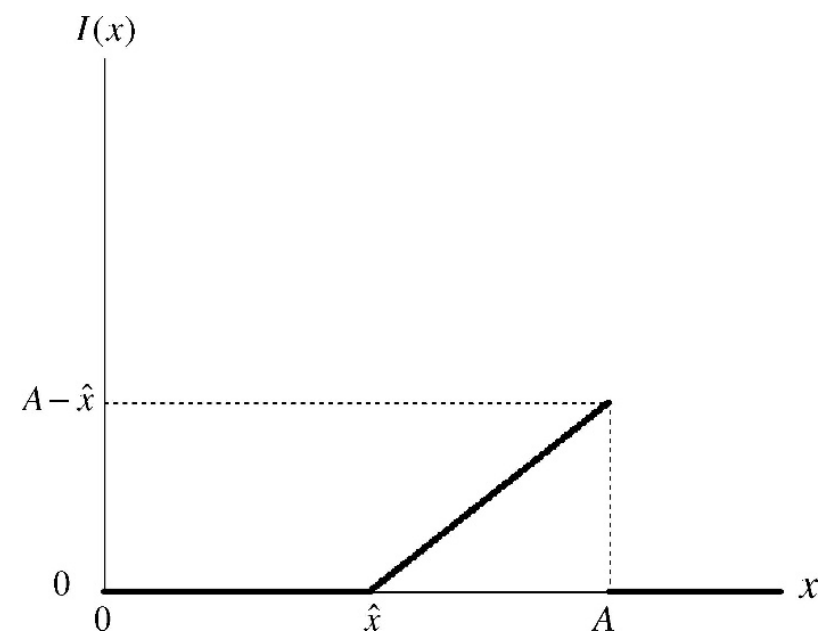

Figure 3. Indemnity versus Loss.

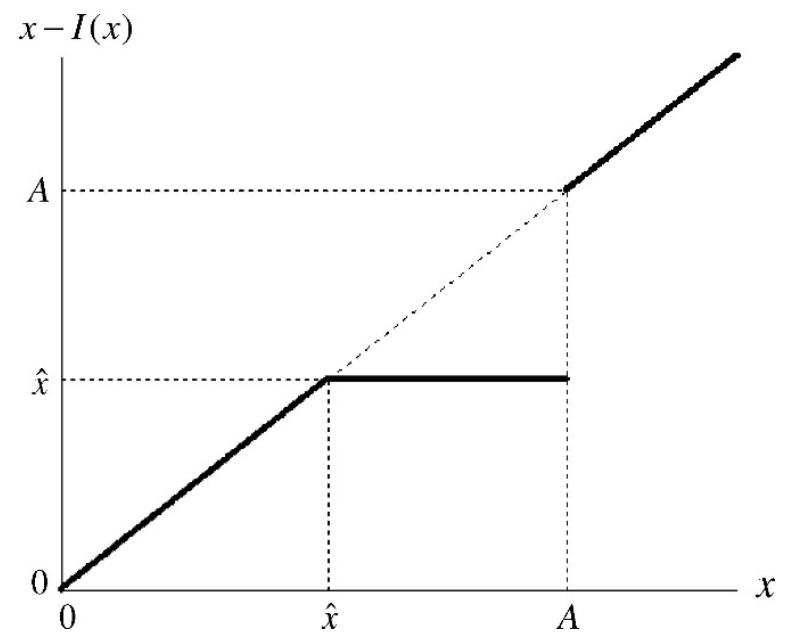

Figure 4. Retention versus Loss.

Assuming insurance is required $(A>\hat{x})$, then based on equation (14), the expected indemnity is decreasing and convex in $v$ and $\alpha$. Accordingly, the expected wealth is increasing and concave in $v$ and $\alpha$, since $\bar{W}=W_{0}-\bar{x}-\lambda \bar{I}$. That is,

$$
\partial \bar{W} / \partial \nu>0>\partial^{2} \bar{W} / \partial v^{2} \quad \text { and } \quad \partial \bar{W} / \partial \alpha>0>\partial^{2} \bar{W} / \partial \alpha^{2} \quad \text { if } A>\hat{x} \text {. }
$$




\subsection{Alternative insurances}

Case 1: Proportional coinsurance policy. Assume the indemnity schedule is limited to coinsurance provision, $I(x)=\theta x$, where $\theta$ denotes the coinsurance proportion. Accordingly, the optimality problem (equation (3)) is revised as follows.

$$
\begin{array}{cl}
\underset{\theta}{\operatorname{Minimize}} & \bar{I}=\theta \bar{x} \\
\text { Subject to } & \operatorname{Pr}\{X-\theta X \leq v+\bar{x}-\bar{I}\} \geq 1-\alpha
\end{array}
$$

From equation (17), we obtain $\theta \geq 1-v /\left[F^{-1}(1-\alpha)-\bar{x}\right]$. Substituting this fact into equation (16) obtains

$$
\begin{aligned}
& \theta=1-v /\left[F^{-1}(1-\alpha)-\bar{x}\right], \\
& \bar{I}=\theta \bar{x}=\left\{1-v /\left[F^{-1}(1-\alpha)-\bar{x}\right]\right\} \bar{x} .
\end{aligned}
$$

Equation (19) implies that the expected indemnity $\bar{I}$ is decreasing and liner in $v$.

Case 2: Deductible policy. Assume the indemnity schedule is limited to deductible clause, $I(x)=\operatorname{Max}\{x-D, 0\}$, where $D$ represents the deductible. Accordingly, the optimality problem (equation (3)) is revised as follows.

$$
\begin{array}{cl}
\underset{D}{\operatorname{Minimize}} & \bar{I}=\int_{D}^{\infty}(x-D) f(x) d x \\
\text { Subject to } & \operatorname{Pr}\{\operatorname{Min}\{X, D\} \leq v+\bar{x}-\bar{I}\} \geq 1-\alpha
\end{array}
$$

Given $\operatorname{Pr}\{X \leq v+\bar{x}\} \geq 1-\alpha$, from equation (5), the insured need not purchase insurance, i.e. $I(x) \equiv 0$ or $D \geq \operatorname{Max}\{x\}$. Meanwhile, given $\operatorname{Pr}\{X \leq v+\bar{x}\}<1-\alpha$, the insured should purchase insurance, that is, $D<\operatorname{Max}\{x\}$. If the deductible $D>v+\bar{x}-\bar{I}$, then equation (21) can be modified as follows.

$$
\operatorname{Pr}\{X \leq v+\bar{x}-\bar{I}\} \geq 1-\alpha
$$

However, equation (22) clearly deviates the condition that $\operatorname{Pr}\{X \leq v+\bar{x}\}<1-\alpha$. Therefore, $D \leq v+\bar{x}-\bar{I}$ in the case that insured need purchase insurance. Taking the constraint $D \leq v+\bar{x}-\bar{I}$ into equation (20), $D=v+\bar{x}-\bar{I}$ since $\bar{I}$ is decreasing in $D$. In sum, the optimal deductible schedule can be arranged as follows.

$$
D \geq \operatorname{Max}\{x\} \quad \text { if } \operatorname{Pr}\{X \leq v+\bar{x}\} \geq 1-\alpha
$$


and

$$
\left\{\begin{array}{l}
D=v+\bar{x}-\bar{I} \\
\bar{I}=\int_{D}^{\infty}(x-D) f(x) d x
\end{array} \quad \text { if } \operatorname{Pr}\{X \leq v+\bar{x}\}<1-\alpha .\right.
$$

Appendix 3 shows that the expected indemnity $\bar{I}$ is decreasing and convex in $v$.

Case 3: Upper-limit policy. Assume the indemnity schedule is limited to $I(x)=\operatorname{Min}\{x$, $M$ \}, where $M$ is the upper-limit. Accordingly, the optimality problem (equation (3)) is revised as follows.

$$
\begin{array}{cl}
\underset{M}{\operatorname{Minimize}} & \bar{I}=\int_{0}^{M} x f(x) d x+\int_{M}^{\infty} M f(x) d x \\
\text { Subject to } & \operatorname{Pr}\{\operatorname{Max}\{X, M\} \leq v+\bar{x}+M-\bar{I}\} \geq 1-\alpha
\end{array}
$$

In the case where $\operatorname{Pr}\{X \leq v+\bar{x}\} \geq 1-\alpha$, from equation (5), the insured need not purchase insurance; that is, $I(x) \equiv 0$ or $M=0$. Meanwhile, when $\operatorname{Pr}\{X \leq v+\bar{x}\}<1-\alpha$, the insured should purchase insurance; that is, $M>0$. Since the inequality $M<v+\bar{x}+M-\bar{I}$ always holds, equation (26) can be modified to

$$
\operatorname{Pr}\{X \leq v+\bar{x}+M-\bar{I}\} \geq 1-\alpha .
$$

From equations (25) and (27),

$$
\int_{0}^{M} x f(x) d x-M F(M)=v+\bar{x}-F^{-1}(1-\alpha),
$$

and

$$
\bar{I}=v+\bar{x}+M-F^{-1}(1-\alpha) .
$$

Appendix 4 shows that the expected indemnity $\bar{I}$ is decreasing and concave in $v$.

\section{Insurance policy under uniform distribution}

Assume $X$ obeys the uniform distribution, with probability density function

$$
f_{U}(x)=1 / h, \quad 0 \leq x \leq h .
$$

Accordingly, the cumulative distribution function

$$
F_{U}(x)=x / h, \quad 0 \leq x \leq h,
$$


and the expected value

$$
\bar{x}=h / 2 \text {. }
$$

For convenience, $U_{a}^{b}$ is defined and calculated as follows.

$$
U_{a}^{b} \equiv \int_{a}^{b} x f_{U}(x) d x=\int_{a}^{b} \frac{x}{h} d x=\frac{b^{2}-a^{2}}{2 h}
$$

From equations (5), (31) and (32), the condition that the loss requires insurance is

$$
v<(0.5-\alpha) h
$$

For convenience, this Section assumes that equation (34) holds.

\subsection{Optimal insurance}

Combining equations (9) and (31) obtains

$$
A=F_{U}^{-1}(1-\alpha)=(1-\alpha) h
$$

From equations (10), (30), (31), and (33),

$$
\int_{\hat{x}}^{A}(x-\hat{x}) f_{U}(x) d x=U_{\hat{x}}^{A}-\hat{x}\left[F_{U}(A)-F_{U}(\hat{x})\right]=\bar{I} .
$$

Substituting $\hat{x}=v+\bar{x}-\bar{I}=v+h / 2-\bar{I}$ and equation (35) into equation (36) yields

$$
\frac{(1-\alpha)^{2} h^{2}-\hat{x}^{2}}{2 h}-\hat{x}\left(\frac{(1-\alpha) h-\hat{x}}{h}\right)=v+\frac{h}{2}-\hat{x}
$$

Solving above equation obtains

$$
\hat{x}=-\alpha h+\sqrt{2 h(v+\alpha h)} \text {. }
$$

Moreover, substituting $\hat{x}=v+h / 2-\bar{I}$ into equation (38) yields

$$
\bar{I}=v+h / 2+\alpha h-\sqrt{2 h(v+\alpha h)}
$$




\subsection{Alternative insurance}

Case 1: Proportional coinsurance policy. Combining equations (18), (31) and (32) can obtain the optimal coinsurance proportion

$$
\theta=1-\frac{v}{F_{U}^{-1}(1-\alpha)-\bar{x}}=1-\frac{v}{(0.5-\alpha) h}
$$

Substituting equations (32) and (40) into equation (19) obtains

$$
\bar{I}=\frac{h}{2}-\frac{v}{1-2 \alpha}
$$

Case 2: Deductible policy. Substituting equations (31) and (32) into equation (24) obtains

$$
\left\{\begin{array}{l}
D=v+\bar{x}-\bar{I} \\
\bar{I}=\int_{D}^{h} \frac{x-D}{h} d x
\end{array}\right.
$$

Simplifying equation (42) yields

$$
\left\{\begin{array}{l}
D=\sqrt{2 v h} \\
\bar{I}=h / 2+v-\sqrt{2 v h}
\end{array}\right.
$$

Case 3: Upper-limit policy. From equations (31), (32) and (33), equation (28) is rewritten as follows.

$$
\frac{M^{2}}{2 h}-\frac{M^{2}}{h}=v+\frac{h}{2}-(1-\alpha) h
$$

Solving the above equation, the optimal upper-limit policy is obtained as follows.

$$
M=h \sqrt{1-2 \alpha-2 v / h} .
$$

Substituting equations (31), (32) and (45) into equation (29) obtains

$$
\bar{I}=h[\sqrt{1-2 \alpha-2 v / h}-0.5+\alpha]+v .
$$




\section{Insurance policy under lignormal distribution}

Assume $\log X$ has a normal distribution with mean $\mu$ and variance $\sigma^{2}$. By definition, $X$ obeys the lognormal distribution with probability density function

$$
f_{L}(x)=\frac{1}{x \sigma \sqrt{2 \pi}} e^{-\frac{1}{2}\left(\frac{\log x-\mu}{\sigma}\right)^{2}}, \quad x \geq 0 .
$$

From equation (47), Appendix 5 shows that the cumulative distribution

$$
F_{L}(x)=\Phi\left(\frac{\log x-\mu}{\sigma}\right), \quad x \geq 0
$$

where $\Phi(\cdot)$ denotes the cumulative standard normal distribution. Additionally, $L_{a}^{b}$ is defined and calculated as follows.

$$
L_{a}^{b} \equiv \int_{a}^{b} x f(x) d x=e^{\mu+\sigma^{2} / 2}\left[\Phi\left(\frac{\log b-\mu}{\sigma}-\sigma\right)-\Phi\left(\frac{\log a-\mu}{\sigma}-\sigma\right)\right] .
$$

Appendix 6 provides the proof of equation (49). From equation (49), the expected value

$$
\bar{x}=L_{0}^{\infty}=e^{\mu+\sigma^{2} / 2} .
$$

From equations (5), (48) and (50), the condition in which insurance is required against a potential loss is

$$
v<e^{\mu+\sigma \Phi^{-1}(1-\alpha)}-e^{\mu+\sigma^{2} / 2}
$$

For convenience, equation (51) is assumed to hold in this Section.

\subsection{Optimal insurance}

Combining equations (9) and (48) obtains

$$
A=F_{L}^{-1}(1-\alpha)=e^{\mu+\sigma \Phi^{-1}(1-\alpha)} .
$$

From equations (10), (48) and (49),

$$
\bar{I}=\int_{\hat{x}}^{A}(x-\hat{x}) f(x) d x=L_{\hat{x}}^{A}-\hat{x}\left[F_{L}(A)-F_{L}(\hat{x})\right] .
$$


Substituting $\bar{I}=v+\bar{x}-\hat{x}$ into equation (53), $\hat{x}$ can be obtained by

$$
L_{\hat{x}}^{A}-\hat{x}\left[1-\alpha-F_{L}(\hat{x})\right]=v+\bar{x}-\hat{x} .
$$

\subsection{Alternative insurance}

Case 1: Proportional coinsurance policy. Combining equations (18), (48) and (50), the optimal coinsurance proportion is

$$
\theta=1-\frac{v}{e^{\mu+\sigma \Phi^{-1}(1-\alpha)}-e^{\mu+\sigma^{2} / 2}}
$$

Substituting equations (50) and (55) into equation (19) produces

$$
\bar{I}=e^{\mu+\sigma^{2} / 2}-\frac{v}{e^{\sigma \Phi^{-1}(1-\alpha)-\sigma^{2} / 2}-1}
$$

Case 2: Deductible policy. Substituting equations (48) and (50) into equation (24) obtains

$$
\left\{\begin{array}{l}
D=v+e^{\mu+\sigma^{2} / 2}-\bar{I} \\
\bar{I}=L_{D}^{\infty}-D\left[1-F_{L}(D)\right]
\end{array}\right.
$$

Case 3: Upper-limit policy. From equations (28), (48), (49) and (50), $M$ can be solved by

$$
L_{0}^{M}-M F_{L}(M)=v+e^{\mu+\sigma^{2} / 2}-e^{\mu+\sigma \Phi^{-1}(1-\alpha)}
$$

Substituting equations (48), (50) and (59) into equation (29) obtains

$$
\bar{I}=v+e^{\mu+\sigma^{2} / 2}-e^{\mu+\sigma \Phi^{-1}(1-\alpha)}+M .
$$

\section{Conclusions}

This study derives the optimal insurance policy form endogenously under a VaR framework. In addition to deriving the optimal insurance, this study also calculates the optimal insurance levels for three alternative insurances. We show that the optimal insurance policy can be replicated by three options, including a long call option with a small strike price, a short call option with a large strike price, and a short cash-or-nothing call option. Additionally, the main comparative static results are as follows.

For the optimal insurance and the deductible policy, the expected indemnity is decreasing and convex in VaR. Next, for the proportional coinsurance policy, the expected indemnity is decreasing and linear in VaR. Finally, for the upper-limit policy, the expected indemnity is decreasing and concave in VaR. 
Following previous investigations, this study assumes that the loading percentage is fixed relative to the expected indemnity. Although this assumption frequently is consistent with insurance practices, differences exist in regular loading fees. For example, the loading percentage can be increasing or decreasing in the expected indemnity. If the loading percentage is not fixed, then the provided optimal insurance form may be revised. Consequently, future research can attempt to develop the optimal insurance form with the variable loading percentage case.

\section{Appendix 1: Prove that if $A>\hat{x}$ then $\partial^{2} \bar{I} / \partial \nu^{2}>0>\partial \bar{I} / \partial \nu$ for the optimal insurance}

Partially differentiating $\hat{x} \equiv v+\bar{x}-\bar{I}$ in equation (10) with respect to $v$ yields

$$
\partial \hat{x} / \partial v=1-\partial \bar{I} / \partial \nu .
$$

Fixed $\alpha$ (i.e., $A$ is fixed) and partially differentiating equation (10) with respect to $v$ yields

$$
-\frac{\partial \hat{x}}{\partial v}(\hat{x}-\hat{x}) f(\hat{x})+\int_{\hat{x}}^{A}-\frac{\partial \hat{x}}{\partial v} f(x) d x=\frac{\partial \bar{I}}{\partial v} .
$$

Simplified equation (A2) yields

$$
-[\partial \hat{x} / \partial \nu][F(A)-F(\hat{x})]=\partial \bar{I} / \partial \nu .
$$

Substituting equation (A1) into equation (A3) yields

$$
[\partial \bar{I} / \partial \nu]\{1-[F(A)-F(\hat{x})]\}=-[F(A)-F(\hat{x})] .
$$

Partially differentiating equation (A4) with respect to $v$ yields

$$
\left[\partial^{2} \bar{I} / \partial v^{2}\right]\{1-[F(A)-F(\hat{x})]\}+[\partial \bar{I} / \partial \nu]\{f(\hat{x})[\partial \hat{x} / \partial \nu]\}=f(\hat{x})[\partial \hat{x} / \partial \nu] .
$$

Substituting equation (A1) into equation (A5) yields

$$
\left[\partial^{2} \bar{I} / \partial \nu^{2}\right]\{1-[F(A)-F(\hat{x})]\}=f(\hat{x})[\partial \hat{x} / \partial \nu]^{2} .
$$

Since $A>\hat{x}, 0<[F(A)-F(\hat{x})]<1$. Incorporating this fact into both equations (A4) and (A6) produces $\partial^{2} \bar{I} / \partial v^{2}>0>\partial \bar{I} / \partial v$.

\section{Appendix 2: Prove that if $A>\hat{x}$ then $\partial^{2} \bar{I} / \partial \alpha^{2}>0>\partial \bar{I} / \partial \alpha$} for the optimal insurance

Since $F(A)=1-\alpha$, differentiating this equation with respect to $\alpha$ can yield

$$
[\partial A / \partial \alpha] f(A)=-1 \text {. }
$$


Partially differentiating $\hat{x} \equiv v+\bar{x}-\bar{I}$ in equation (10) with respect to $\alpha$ yields

$$
\partial \hat{x} / \partial \alpha=-\partial \bar{I} / \partial \alpha
$$

Partially differentiating equation (10) with respect to $\alpha$ yields

$$
\frac{\partial A}{\partial \alpha}(A-\hat{x}) f(A)-\frac{\partial \hat{x}}{\partial \alpha}(\hat{x}-\hat{x}) f(\hat{x})+\int_{\hat{x}}^{A}-\frac{\partial \hat{x}}{\partial \alpha} f(x) d x=\frac{\partial \bar{I}}{\partial \alpha} .
$$

Substituting equations (A7) and (A8) into equation (A9) yields

$$
[\partial \bar{I} / \partial \alpha]\{1-[F(A)-F(\hat{x})]\}=-(A-\hat{x}) .
$$

Partially differentiating equation (A10) with respect to $\alpha$ yields

$$
\frac{\partial^{2} \bar{I}}{\partial \alpha^{2}}(1-[F(A)-F(\hat{x})])+\frac{\partial \bar{I}}{\partial \alpha}\left[1+f(\hat{x}) \frac{\partial \hat{x}}{\partial \alpha}\right]=-\frac{\partial A}{\partial \alpha}+\frac{\partial \hat{x}}{\partial \alpha}
$$

Substituting equation (A8) into equation (A11) yields

$$
\left(\partial^{2} \bar{I} / \partial \alpha^{2}\right)(1-[F(A)-F(\hat{x})])=f(\hat{x})(\partial \bar{I} / \partial \alpha)^{2}-(\partial A / \partial \alpha)-2(\partial \bar{I} / \partial \alpha) .
$$

Since $A>\hat{x}, 0<[F(A)-F(\hat{x})]<1$. Incorporating this fact into equation (A10) yields $\partial \bar{I} / \partial \alpha<0$. Additionally, $\partial A / \partial \alpha<0$ by equation (A7), $F(A)-F(\hat{x})<1$, and $\partial \bar{I} / \partial \alpha<0$, thus $\partial^{2} \bar{I} / \partial v^{2}>0$.

Appendix 3: Proof that if $A>\hat{x}$ then $\partial \bar{I} / \partial \nu<0$ and $\partial^{2} \bar{I} / \partial \nu^{2}>0$ for the deductible policy.

From equation (24), we have

$$
\bar{I}=\int_{D}^{\infty}[x-(v+\bar{x}-\bar{I})] f(x) d x
$$

Substituting the fact of $D=v+\bar{x}-\bar{I}$ from equation (24) into equation (A13) yields

$$
\bar{I}=\int_{v+\bar{x}-\bar{I}}^{\infty} x f(x) d x-(v+\bar{x}-\bar{I})[1-F(D)]
$$

Partially differentiating equation (A14) with respect to $v$ yields

$$
\frac{\partial \bar{I}}{\partial v}=-\left[1-\frac{\partial \bar{I}}{\partial v}\right] D f(D)-\left[1-\frac{\partial \bar{I}}{\partial v}\right][1-F(D)]+D f(D)\left[1-\frac{\partial \bar{I}}{\partial v}\right]
$$


Simplifying equation (A15) yields

$$
(\partial \bar{I} / \partial v) F(D)=-[1-F(D)]
$$

Partially differentiating equation (A16) with respect to $v$ yields

$$
\left(\partial^{2} \bar{I} / \partial \nu^{2}\right) F(D)+(\partial \bar{I} / \partial v)(1-\partial \bar{I} / \partial \nu) f(D)=(1-\partial \bar{I} / \partial \nu) f(D)
$$

Simplifying equation (A17) yields

$$
\left(\partial^{2} \bar{I} / \partial \nu^{2}\right) F(D)=[1-\partial \bar{I} / \partial \nu]^{2} f(D)
$$

Since $0<F(D)<1$, equation (A16) states $\partial \bar{I} / \partial v<0$. Additionally, equation (A18) clearly states $\partial^{2} \bar{I} / \partial v^{2}>0$.

Appendix 4: Proof that if $A>\hat{x}$ then $\partial \bar{I} / \partial \nu<0$ and $\partial^{2} \bar{I} / \partial \nu^{2}<0$ for the upper-limit policy

Partially differentiating equation (29) with respect to $v$ yields

$$
\partial \bar{I} / \partial v=1+\partial M / \partial v .
$$

Partially differentiating equation (A19) with respect to $v$ yields

$$
\partial^{2} \bar{I} / \partial v^{2}=\partial^{2} M / \partial v^{2}
$$

Partially differentiating equation (28) with respect to $v$ yields

$$
[\partial M / \partial \nu] M f(M)-[\partial M / \partial \nu] F(M)-M f(M)[\partial M / \partial \nu]=1 .
$$

Simplifying equation (A21) yields

$$
[\partial M / \partial \nu] F(M)=-1 .
$$

Substituting equation (A19) into equation (A22) yields

$$
\partial \bar{I} / \partial v=-[1-F(M)] / F(M) .
$$


Since $0<F(M)<1$, equation (A23) states $\partial \bar{I} / \partial v<0$. Additionally, partially differentiating equation (A22) with respect to $v$ yields

$$
\left[\partial^{2} M / \partial \nu^{2}\right] F(M)+[\partial M / \partial \nu] f(M)[\partial M / \partial \nu]=0 .
$$

Substituting equation (A20) into equation (A24) yields

$$
\partial^{2} I / \partial \nu^{2}=-[\partial M / \partial \nu]^{2} f(M) / F(M)<0 .
$$

\section{Appendix 5: Proof of equation (48)}

By definition,

$$
F_{L}(x) \equiv \int_{0}^{x} \frac{1}{x \sigma \sqrt{2 \pi}} e^{-\frac{1}{2}\left(\frac{\log x-\mu}{\sigma}\right)^{2}} d x
$$

Let $z=(\log x-\mu) / \sigma$, then $d z=d x /(\sigma x)$ and equation (A26) can be rewritten as

$$
F_{L}(x) \equiv \int_{-\infty}^{\frac{\log x-\mu}{\sigma}} \frac{1}{\sqrt{2 \pi}} e^{-z^{2} / 2} d z=\Phi\left(\frac{\log x-\mu}{\sigma}\right)
$$

\section{Appendix 6: Proof of equation (49)}

By definition,

$$
L_{a}^{b} \equiv \int_{a}^{b} \frac{1}{\sigma \sqrt{2 \pi}} e^{-\frac{1}{2}\left(\frac{\log x-\mu}{\sigma}\right)^{2}} d x
$$

Let $z=(\log x-\mu) / \sigma$, then $d x=\sigma e^{\mu+\sigma z} d z$ and equation (A28) can be rewritten as

$$
L_{a}^{b} \equiv \int_{\frac{\log a-\mu}{\sigma}}^{\frac{\log b-\mu}{\sigma}} \frac{1}{\sqrt{2 \pi}} e^{\mu+\sigma z} e^{-z^{2} / 2} d z
$$

Let $y=z-\sigma$, then equation (A29) can be rewritten as

$$
\begin{aligned}
L_{a}^{b} & \equiv e^{\mu+\sigma^{2} / 2} \int_{\frac{\log a-\mu}{\sigma}-\sigma}^{\frac{\log b-\mu}{\sigma}-\sigma} \frac{1}{\sqrt{2 \pi}} e^{-y^{2} / 2} d y \\
& =e^{\mu+\sigma^{2} / 2}\left[\Phi\left(\frac{\log b-\mu}{\sigma}-\sigma\right)-\Phi\left(\frac{\log a-\mu}{\sigma}-\sigma\right)\right] .
\end{aligned}
$$




\section{Acknowledgments}

We are grateful to the editor and Professor Harris Schlesinger for many helpful comments and instructions. Especially, Professor Harris Schlesinger suggested a more refined and straightforward approach for the optimal insurance design. This refinement is applied in Section 2.2. of this paper.

\section{Notes}

1. As described by Dowd [1999] and Jorion [2001], VaR is being adopted by investment banks, commercial banks, pension funds, other financial institutions, regulators, asset managers, and non-financial corporations.

2. Campbell et al. [2001] developed a portfolio selection model that allocates financial assets by maximizing expected returns subject to the constraint that expected maximum loss should meet the VaR limits. Basak and Shapiro [2001] found an optimal dynamic portfolio for utility maximizing investors, who must use VaR to manage risk exposure.

3. A Pareto efficient allocation is one for which there is no way to make all agents better off (Varian [1992], p. 225). Hence, a Pareto efficient allocation is one that maximizes the satisfaction of the insured, while holding the insurer fixed at some given satisfaction. In this study, the insurer's satisfaction is unchanged if the insurance premium is $P=(1+\lambda) \bar{I}$.

4. Once the insured selects some sub-interval of $x>v+\bar{x}-\bar{I}$ to insure, to save loading fees, the retention, $x-I(x)$, on the sub-interval must equal $v+\bar{x}-\bar{I}$.

\section{References}

ARROW, K.J. [1974]: “Optimal Insurance and Generalized Deductibles,” Scandinavian Actuarial Journal, 1, $1-42$.

BASAK, S. and SHAPIRO, A. [2001]: "Value-at-Risk-Based Risk Management: Optimal Policies and Asset Prices," Review of Financial Studies, 14, 371-405.

BORCH, K. [1960]: “The Safety Loading of Reinsurance Premium,” Skandinavian Aktuarietidskrift, 162-84.

CAMPBELL, R. HUISMAN, R., and KOEDIJK, K. [2001]: "Optimal Portfolio Selection in a Value at Risk Framework," Journal of Banking and Finance, 25, 1789-1804.

DOHERTY, N.A. and EECKHOUDT L. [1995]: "Optimal Insurance Without Expected Utility: The Dual Theory and the Linearity of Insurance Contracts," Journal of Risk and Uncertainty, 10, 157-179.

DOHERTY, N.A. and SCHLESINGER, H. [1983a]: "Optimal Insurance in Incomplete Markets," Journal of Political Economy, 91, 1045-1054.

DOHERTY, N.A. and SCHLESINGER, H. [1983b]: "The Optimal Deductible for an Insurance Policy When Initial Wealth is Random," Journal of Business, 56, 555-565.

DOWD, K. [1999]: Beyond Value at Risk: The New Science of Risk Management. John Wiley \& Sons Ltd.

GOLLIER, C. [1987]: "The Design of Optimal Insurance Contracts Without the Nonnegativity Constraint on Claims," Journal of Risk and Insurance, 54, 314-324.

GOLLIER, C. [1996]: “Optimal Insurance of Approximate Losses,” Journal of Risk and Insurance, 63, 369-380. GOLLIER, C. [2003]: "To Insure or Not to Insure?: An Insurance Puzzle," The Geneva Papers on Risk and Insurance Theory, 28, 5-24.

GOLLIER, C. and SCHLESINGER, H. [1996]: “Arrow's Theorem on the Optimality of Deductibles: A Stochastic Dominance Approach,” Economic Theory, 7, 359-363.

HUBERMAN, G. MAYERS, D., and SMITH, C.W. [1983]: "Optimal Insurance Policy Indemnity Schedules," Bell Journal of Economics, 14, 415-426.

HULL, C. J. [2003]: Options, Futures, and Other Derivatives, 5e, Prentice Hall.

JORION, P. [2001]: Value at Risk: The New Benchmark for Managing Financial Risk, McGraw-Hill International Edition. 
LI, C.S. and LIU, C.C. [2003]: “On Compulsory Per-Claim Deductibles in Automobile Insurance,” The Geneva Papers on Risk and Insurance Theory, 28, 25-32.

RAVIV, A. [1979]: “The Design of an Optimal Insurance Policy,” American Economic Review, 69, 84-96.

SCHLESINGER, H. [1981]: "The Optimal Level of Deductibility in Insurance Contracts," Journal of Risk and Insurance, 48, 465-481.

SCHLESINGER, H. [1997]: "Insurance Demand Without the Expected-Utility Paradigm,” Journal of Risk and Insurance, 64, 19-39.

VARIAN, H.R. [1992]: Microeconomic Analysis, 3e, W. W. Norton \& Company, Inc. 